\title{
Occurrence, Distribution, and Molecular Characterization of Citrus yellow vein clearing virus in China
}

Y. Zhou, H. M. Chen, M. J. Cao, X. F. Wang, X. Jin, K. H. Liu, and C. Y. Zhou, Citrus Research Institute, Southwest University, Chongqing, 400712, P. R. China

\begin{abstract}
In 2009, a new citrus viral disease caused by Citrus yellow vein clearing virus (CYVCV) was discovered in China. To more effectively monitor the presence of CYVCV, a survey was conducted in 166 citrus orchards from 11 major citrus-growing provinces in China from May 2014 to April 2016. In all, 458 of a total of 2,350 citrus samples tested positive for CYVCV, demonstrating that the virus is widely distributed in China. In this study, the complete genome sequences of $19 \mathrm{CYVCV}$ isolates from different provinces and hosts were sequenced and characterized. Comparisons of the whole-genome sequences of these 19

CYVCV isolates as well as 4 isolates previously reported from around the world revealed that the sequence identity ranged from 97.1 to $99.8 \%$, indicating that there is a very low level of sequence heterogeneity among CYVCV isolates of different geographic origins and hosts. Phylogenetic analysis of these 23 genomic sequences suggested that all of the isolates from China were clustered into the same clade, clearly apart from the CYVCV isolates from Turkey and Pakistan. To our knowledge, this is the first extensive survey conducted in China for CYVCV incidence.
\end{abstract}

Yellow vein clearing disease (YVCD) of citrus, an emerging viral disease, was first reported from Pakistan in 1988 in lemon (Citrus limon Burm. f.) and sour orange (C. aurantium L.) (Catara et al. 1992). Symptoms in affected lemon and sour orange included yellow vein clearing, leaf distortion, and water soaking of veins on the ventral side. The same symptoms were also reported on Etrog citron (C. medica L.), Rangpur lime (C. limonia Osbeck), sour orange, and some varieties of lemon in Turkey and India (Alshami et al. 2003; Önelge 2002).

Citrus yellow vein clearing virus (CYVCV), a recently described member of the genus Mandarivirus, family Alphaflexiviridae, is considered the causal agent of YVCD (Loconsole et al. 2012a). The viral genome consists of a single-stranded positive-sense RNA molecule of around $7.5 \mathrm{~kb}$, with six predicted open reading frames (ORF) (Loconsole et al. 2012a). CYVCV is transmitted through vegetative propagation of infected buds, scion, or rootstocks and by mechanical inoculations of sap extracts onto herbaceous indicator hosts (Alshami et al. 2003; Önelge et al. 2011). CYVCV can also be transmitted by Aphis craccivora and A. spiraecola from lemon to bean (Phaseolus vulgaris var. Dermason), and from bean to bean (Önelge 2002; Önelge et al. 2011). No additional ways of transmission are known (Zhou et al. 2015).

In 2009, the typical symptoms caused by CYVCV were first observed on 'Eureka' lemon (C. limon Burm. f.) in Ruili, Yunnan Province of China (Chen et al. 2014), followed by Anyue, Sichuan Province, where more than $80 \%$ of Chinese lemon production is concentrated (Zhou et al. 2016). Because this virus severely hinders the growth and fruit yield of Eureka lemon, it is of economical importance (Chen et al. 2014; Zhou et al. 2016). Until now, the only known way to slow down its dissemination was through the national scheme for the production of certified citrus planting material (Loconsole et al. 2012a). Unfortunately, due to its unknown distribution in orchards and nurseries and the absence of any risk analysis addressing its potential impact on other citrus cultivars in China, CYVCV is not included in the national citrus certification scheme. Recent sequence analysis of three full-length sequences of CYVCV isolated in lemon from China, Pakistan,

Corresponding author: C. Y. Zhou; E-mail: zhoucy@cric.cn

Accepted for publication 17 August 2016.

C) 2017 The American Phytopathological Society and Turkey indicated that there existed genetic stability among CYVCV isolates (Cao et al. 2016; Zhen et al. 2015). Nevertheless, further studies are needed to get a more complete overview of the CYVCV population structure worldwide. The present study was undertaken to provide further insight on the distribution of CYVCV on citrus in China. In addition, the full sequences of $19 \mathrm{CYVCV}$ isolates from 11 major citrus-growing provinces of China were used to produce a thorough molecular variability analysis.

\section{Materials and Methods}

The field survey. The field survey and collection of samples were conducted from May 2014 to April 2016 in the 11 major citrus-growing provinces of China, including Sichuan, Chongqing, Yunnan, Guizhou, Guangxi, Guangdong, Hubei, Hunan, Fujian, Jiangxi, and Zhejiang. In total, 2,350 citrus samples were collected from 166 orchards.

Virus symptoms observed in orchards. The samples were randomly evaluated both for normal appearance and virus-like symptoms of leaf distortion, vein clearing, and chlorosis in each orchard. The relationship between reverse-transcription polymerase chain reaction (RT-PCR) results and symptoms was studied by careful observation of leaf symptoms at every sampling date. Furthermore, the samples with symptoms were graft inoculated onto citrus indicator Eureka lemon. The same indicator was mock inoculated with virus-free blind buds as a control. All plants were maintained in an insect-proof greenhouse at 20 to $25^{\circ} \mathrm{C}$. Symptoms were monitored throughout the year. These plants were screened by RT-PCR or PCR for the presence of CYVCV, Citrus tristeza virus (CTV), Citrus tatter leaf virus (CTLV), Satsuma dwarf virus (SDV), Citrus chlorotic dwarf-associated virus $(\mathrm{CCDaV})$, Citrus vein enation virus (CVEV) (Iwanami et al. 1998; Loconsole et al. 2012a,b; Roy et al. 2005; Vives et al. 2013), and six known citrus viroids (Ito et al. 2002).

Nucleic acid extraction and RT-PCR detection. Total RNA extracts were obtained from each plant with Trizol reagent (Invitrogen, Carlsbad, CA), and used for one-step RT-PCR detection with the specific primers VF-1/VR-1, as previously described (Chen et al. 2015).

Cloning and sequencing. For cloning, $19 \mathrm{CYVCV}$-positive samples were randomly selected from different citrus cultivars and geographical origins (Table 1). Three sets of overlapping primers (1fw/2652rew, 2526f/5771r, and 5638f/7531r) were used for genome amplification based on CYVCV genome sequence (KP313240) available in GenBank (Table 2). The $5^{\prime}$ and $3^{\prime}$ termini were 
determined by the SMARTer RACE cDNA Amplification Kit (Clontech, Mountain View, CA) using the specific primers 7081fw/921 rev and an oligo(dT) primer, as previously described (Loconsole et al. 2012a). PCR amplicons were purified and cloned into pGEM-T easy vector (Promega Corp., Madison, WI). Five clones per fragment were custom sequenced (Beijing Genomics Institute, Shengzhen, GD, China). Complete genome sequences were assembled using BioEdit 7.0.5.3 and deposited in GenBank under accession numbers KX156734 to KX156752.

Genetic diversity and phylogenetic analyses. Multiple nucleotide sequence alignments of the complete genome of the 19 CYVCV isolates from this study plus CYVCV-CQ (KP313240), CYVCV-PK(KP313241), CYVCV-YN (KP313242), CYVCV$\mathrm{Y} 1(\mathrm{JX} 040635)$, Indian citrus ringspot virus-K1 (ICRSV-KI, NC003093), ICRSV-Pu (HQ324250), and Potato virus X (PVX, M72416) were conducted separately with CLC Genomics Workbench 8.5.1.

Phylogenetic analysis of the genomic sequences, putative viral replication-associated polyprotein-replicase (REP), and coat protein (CP) was performed with the MEGA analysis package, version 6.0 (Tamura et al. 2013) by using the neighbor-joining (NJ), unweighted pair-group method with arithmetic means (UPGMA), and maximum-likelihood (PAUP*) methods with the JTT matrix and pairwise gap deletion, with 10,000 bootstrap replicates as the test of phylogeny. The cut-off value for the condensed tree was $75 \%$.

\section{Results}

Field survey. CYVCV was detected in $19.5 \%$ of the samples collected in China (458 samples were positive out of the 2,350 samples tested; Table 3). CYVCV was detected in Sichuan, Jiangxi, Guangxi, Guangdong, Hunan, Yunnan, Chongqing, Guizhou, and Fujian Provinces, with an incidence of $41.8 \%$ (77 of 184), 29.4\% (65 of 221), $29.1 \%$ ( 73 of 251 ), $27.5 \%$ ( 50 of 182 ), $26.9 \%$ (65 of 242 ), $21.4 \%$ (59 of 275 ), $8.4 \%$ (65 of 770 ), $7.1 \%$ ( 2 of 28 ), and $2.1 \%$ ( 2 of 96 ), respectively. CYVCV was not found in Hubei ( 0 of 25$)$ and Zhejiang ( 0 of 76) Provinces. In Guangxi, Guangdong, Hunan, Jiangxi, and Yunnan Provinces with a high incidence of CYVCV, the virus could be detected in most of the surveyed orchards. However, in Sichuan Province, CYVCV was found in only 6 of 18 orchards surveyed, and all of them were located in Ziyang and Meishan Counties.

In surveyed citrus cultivars, the most frequently infected cultivar was 'Nanfengmiju' (C. reticulata Blanco), with an infection rate of $68.8 \%$, followed by 'Jiaogan' ( $C$. tankan Hayata, 54.8\%), 'Hongjiangcheng' (C. sinensis (L.) Osbeck, 53.3\%), Eureka lemon (43.8\%), 'Shatangju' (C. reticulata, 34.7\%), 'Gonggan' (C. reticulata Blanco, 28.0\%), 'Bingtangcheng' (C. sinensis (L.) Osbeck, 25.9\%), 'Satsuma' (C. unshiu Marc., 19.6\%), Newhall (C. sinensis (L.) Osbeck navel, 16.9\%),

Table 1. Accession numbers, host, and collection area of Citrus yellow vein clearing virus (CYVCV) isolates used in this study for molecular variability analysis

\begin{tabular}{|c|c|c|c|c|c|c|}
\hline Origin & Isolate & Host & Symptoms $^{\mathrm{a}}$ & Length (bp) & Accession number & Reference \\
\hline \multirow[t]{2}{*}{$\overline{\text { Sichuan }}$} & SC-EL & Eureka lemon & Severe YVC, WC, and LD & 7,529 & KX156748 & This work \\
\hline & SC-NH & Newhall navel orange & No symptoms & 7,529 & KX156749 & This work \\
\hline \multirow[t]{3}{*}{ Chongqing } & CQ-PO & Zaoyangxiaoye trifoliate & No symptoms & 7,529 & KX156735 & This work \\
\hline & CQ-TA & Tarocco blood orange & No symptoms & 7,529 & KX156736 & This work \\
\hline & CYVCV-CQ & Eureka lemon & Severe YVC, WC and LD & 7,531 & KP313240 & Cao et al. 2016 \\
\hline \multirow[t]{4}{*}{ Yunnan } & YN-NH & Newhall navel orange & No symptoms & 7,529 & KX156752 & This work \\
\hline & YN-BTC & $\begin{array}{l}\text { Bingtangcheng common } \\
\text { sweet orange }\end{array}$ & No symptoms & 7,529 & KX156750 & This work \\
\hline & YN-EL & Eureka lemon & Severe YVC, WC, and LD & 7,529 & KX156751 & This work \\
\hline & CYVCV-YN & Eureka lemon & Severe YVC, WC, and LD & 7,529 & KP313242 & Cao et al. 2016 \\
\hline \multirow[t]{2}{*}{ Jiangxi } & JX-NH & Newhall navel orange & No symptoms & 7,529 & KX156747 & This work \\
\hline & JX-NF & Nanfengmiju tangerine & No symptoms & 7,531 & KX156746 & This work \\
\hline \multirow[t]{2}{*}{ Hunan } & HN-GXP & Guanximiyou pummelo & Moderate VC and chlorosis & 7,531 & KX156744 & This work \\
\hline & HN-STJ & Shatangju tangerine & Severe leaf curling & 7,529 & KX156745 & This work \\
\hline \multirow[t]{3}{*}{ Guanxi } & GX-STJ & Shatangju tangerine & Severe leaf curling & 7,529 & KX156742 & This work \\
\hline & GX-GXP & Guanximiyou pummelo & Moderate VC and chlorosis & 7,531 & KX156741 & This work \\
\hline & GX-SA & Satsuma & Mild VC & 7,529 & KX156734 & This work \\
\hline \multirow[t]{3}{*}{ Guangdong } & GD-STP & Shatianyou pummelo & No symptoms & 7,531 & KX156740 & This work \\
\hline & GD-STJ & Shatangju tangerine & Severe leaf curling & 7,529 & KX156739 & This work \\
\hline & GD-JG & Jiaogan mandarin & No symptoms & 7,531 & KX156738 & This work \\
\hline Fujian & FJ-PK & Ponkan & No symptoms & 7,531 & KX156737 & This work \\
\hline Guizhou & GZ-GXP & Guanximiyou pummelo & Moderate VC and chlorosis & 7,531 & KX156743 & This work \\
\hline Pakistan & CYVCV-PK & Sweet orange & No symptoms & 7,529 & KP313241 & Cao et al. 2016 \\
\hline Turkey & CYVCV-Y1 & Kütdiken lemon & Severe YVC, WC, and LD & 7,529 & JX040635 & Zhen et al. 2015 \\
\hline \multicolumn{7}{|c|}{$\begin{array}{l}\text { a Symptoms: Severe yellow vein clearing (YVC), water soaking (WC), and leaf distortion (LD); Moderate vein cleaning (VC) and chlorosis on young leafs of } \\
\text { spring flush; and Mild VC on young leafs of spring flush. }\end{array}$} \\
\hline \multicolumn{2}{|l|}{ Primer } & \multicolumn{2}{|l|}{ Primer sequence } & Product size ( & \multicolumn{2}{|r|}{ Source reference } \\
\hline$\overline{\mathrm{VF}-1}$ & \multicolumn{3}{|c|}{ TACCGCAGCTATCCATTTCC } & 614 & \multicolumn{2}{|r|}{ Chen et al. 2015} \\
\hline VR-1 & \multicolumn{3}{|c|}{ GCAGAAAT CCCGAACCACTA } & & \multicolumn{2}{|r|}{ Chen et al. 2015} \\
\hline $1 \mathrm{fw}$ & \multicolumn{3}{|c|}{ GAAAAGCAAACAKWAHCAWACACACCC } & 2,652 & \multicolumn{2}{|r|}{$\begin{array}{l}\text { Chen et al. } 2015 \\
\text { Loconsole et al } 2012\end{array}$} \\
\hline 2652rew & \multicolumn{3}{|c|}{ GTAATGCGTGATGGGCCATATGAGA } & & \multicolumn{2}{|r|}{ Loconsole et al. 2012a } \\
\hline $2526 \mathrm{f}$ & \multicolumn{3}{|c|}{ TCTCTGCCATCCAAGCACTCCCT } & 3,245 & \multicolumn{2}{|r|}{ This study } \\
\hline $5771 \mathrm{r}$ & \multicolumn{3}{|c|}{ TGAAAGTGAGCAGTGCTAAGGCGGCG } & & \multicolumn{2}{|r|}{ This study } \\
\hline $5638 \mathrm{f}$ & \multicolumn{3}{|c|}{ ACTGCAGATACCCTCACCAAAGAGA } & 1,893 & \multicolumn{2}{|r|}{ This study } \\
\hline $7531 \mathrm{r}$ & \multicolumn{3}{|c|}{ CAGAAATGGAAACTGAAAGC } & & \multicolumn{2}{|r|}{ This study } \\
\hline $7081 \mathrm{fw}$ & \multicolumn{3}{|c|}{ ACCTCACGATGGACCACGTT } & & & Loconsole et al. $2012 \mathrm{a}$ \\
\hline $921 \mathrm{rev}$ & & CAAGAGCACTTGGGTA & & & & Loconsole et al. 2012a \\
\hline
\end{tabular}


and 'Zaoyangxiaoye' trifoliate (Poncirus trifoliate Swingle var. Zaoyangxiaoye, 16.7\%). The incidence of CYVCV in 'Ponkan' (C. reticulata Blanco), 'Tarocco' (C. sinensis (L.) Osbeck), pummelo (C. grandis (L.) Osbeck), and tangor (C. reticulata Blanco $\times C$. sinensis (L.) Osbeck) was less than $10 \%$. CYVCV was not found on 'Bendizao' (C. succosa Hort. ex Tanaka), 'Fengwan' (C. sinensis (L.) Osbeck), 'Cara cara' (C. sinensis (L.) Osbeck), 'Gongshuibaiyou' (C. maxima (L.) Osbeck), 'Shiranui' (C. reticulata Blanco $\times$ C. sinensis (L.) Osbeck), and kumquat (Fortunella sp.). In this study, more than 20 citrus cultivars were autochthonous, and about 10 cultivars were imported from Japan, the United States, Israel, Peru, and Italy. There was no clear correlation between the incidence of CYVCV and the origin of citrus cultivars.

Virus symptoms observed in orchards. Although typical YVCD leaf symptoms such as yellow vein clearing, chlorosis, leaf distortion, and water soaking of veins on the ventral side were observed on CYVCV-infected Eureka lemon and 'Or' (C. reticulata Blanco $\times$
C. sinensis (L.) Osbeck) throughout the year, the symptoms were reduced in hot weather. The young spring flushes of CYVCV-infected Satsuma mandarin and 'Guanximiyou' pummelo (C. grandis (L.) Osbeck) expressed mild vein clearing, moderate vein clearing, and chlorosis, respectively. However, these symptoms were reduced and even disappeared after the leaf matured. Furthermore, these symptoms could not be observed as well during the summer, autumn, and late autumn flushes of the same plant with normal growth. Almost all CYVCV-infected Shatangju plants exhibited severe leaf curling and shrinking during the whole year but those symptoms were also observed in some of Shatangju plants in which CYVCV was not detected. These symptomatic samples collected from Or, Satsuma, Guanximiyou pummelo, and Shatangju were then graft inoculated onto Eureka lemon seedlings. One year postinoculation, severe distortion, strong yellow blotchy chlorosis, and crinkling were observed on young leaves of Eureka lemon. These samples were positive for CYVCV but were free from CTV, CTLV, SDV, CCDaV, CVEV, and viroids,

Table 3. Incidence of Citrus yellow vein clearing virus (CYVCV) in citrus from China

\begin{tabular}{|c|c|c|c|c|c|c|c|c|c|c|c|c|c|}
\hline \multirow[b]{2}{*}{$\begin{array}{l}\text { Genotype, } \\
\text { cultivar }\end{array}$} & \multirow[b]{2}{*}{ Origb } & \multicolumn{11}{|c|}{ Rate of CYVCV in provinces (number of orchards) ${ }^{a}$} & \multirow[b]{2}{*}{ Total $(\%)$} \\
\hline & & $\begin{array}{l}\text { Cho } \\
\text { (41) }\end{array}$ & $\begin{array}{c}\text { Sic } \\
(18)\end{array}$ & $\begin{array}{l}\text { Yun } \\
\text { (15) }\end{array}$ & $\begin{array}{l}\text { Gui } \\
\text { (5) }\end{array}$ & $\begin{array}{l}\text { Zhe } \\
\text { (4) }\end{array}$ & $\begin{array}{l}\text { Fuj } \\
\text { (4) }\end{array}$ & $\begin{array}{l}\text { Gud } \\
\text { (17) }\end{array}$ & $\begin{array}{l}\text { Gux } \\
(21)\end{array}$ & $\begin{array}{l}\text { Jia } \\
\text { (23) }\end{array}$ & $\begin{array}{l}\text { Hun } \\
\text { (15) }\end{array}$ & $\begin{array}{c}\text { Hub } \\
\text { (3) }\end{array}$ & \\
\hline \multicolumn{14}{|l|}{ Lemon } \\
\hline Eureka & US30 & $53 / 223$ & $69 / 87$ & $37 / 53$ & $\ldots$ & $\ldots$ & $\ldots$ & $\ldots$ & $\ldots$ & $\ldots$ & $\ldots$ & $\ldots$ & $159 / 363(43.8)$ \\
\hline \multicolumn{14}{|l|}{ Tangerine } \\
\hline Nanfengmiju & Auto & $0 / 5$ & $\ldots$ & $\ldots$ & $\ldots$ & $\ldots$ & $\ldots$ & $\ldots$ & $\ldots$ & $11 / 11$ & $\ldots$ & $\ldots$ & $11 / 16(68.8)$ \\
\hline Bendizao & Auto & $\ldots$ & $\ldots$ & $\ldots$ & $\ldots$ & $0 / 38$ & $\ldots$ & $\ldots$ & $\ldots$ & $\ldots$ & $\ldots$ & $\ldots$ & $0 / 38(0)$ \\
\hline Shatangju & Auto & $\ldots$ & $\ldots$ & $7 / 41$ & $\ldots$ & $\ldots$ & $\ldots$ & $14 / 70$ & $45 / 75$ & $\ldots$ & $17 / 53$ & $\ldots$ & $83 / 239$ (34.7) \\
\hline Hongju & Auto & $0 / 71$ & $\ldots$ & $\ldots$ & $\ldots$ & $\ldots$ & $\ldots$ & $\ldots$ & $\ldots$ & $\ldots$ & $\ldots$ & $\ldots$ & $0 / 71(0)$ \\
\hline Ponkan & Jpn50 & $0 / 11$ & $\ldots$ & $\ldots$ & $\ldots$ & $\ldots$ & $2 / 42$ & $\ldots$ & $\ldots$ & $\ldots$ & $\ldots$ & $\ldots$ & $2 / 53(3.8)$ \\
\hline \multicolumn{14}{|l|}{ Mandarin } \\
\hline Jiaogan & Auto & $\ldots$ & $\ldots$ & $\ldots$ & $\ldots$ & $\ldots$ & $\ldots$ & $17 / 31$ & $\ldots$ & $\ldots$ & $\ldots$ & $\ldots$ & $17 / 31(54.8)$ \\
\hline Chazhigan & Auto & $\ldots$ & $\ldots$ & $\ldots$ & $\ldots$ & $\ldots$ & $\ldots$ & $0 / 15$ & $\ldots$ & $\ldots$ & $\ldots$ & $\ldots$ & $0 / 15(0)$ \\
\hline Gonggan & Auto & $\ldots$ & $\ldots$ & $\ldots$ & $\ldots$ & $\ldots$ & $\ldots$ & $\ldots$ & $7 / 25$ & $\ldots$ & $\ldots$ & $\ldots$ & $7 / 25(28.0)$ \\
\hline Satsuma & Jpn50 & $0 / 24$ & $0 / 11$ & $0 / 32$ & $0 / 12$ & $0 / 31$ & $0 / 11$ & $\ldots$ & $15 / 15$ & $\ldots$ & $21 / 48$ & $\ldots$ & $36 / 184(19.6)$ \\
\hline \multicolumn{14}{|l|}{ Navel orange } \\
\hline Newhall & US70 & $0 / 34$ & $4 / 21$ & $5 / 86$ & $0 / 5$ & $\ldots$ & $\ldots$ & $2 / 15$ & $0 / 30$ & $54 / 188$ & $1 / 12$ & $\ldots$ & 66/391 (16.9) \\
\hline Fengwan & Auto & $0 / 25$ & $\ldots$ & $\ldots$ & $\ldots$ & $\ldots$ & $\ldots$ & $\ldots$ & $\ldots$ & $\ldots$ & $\ldots$ & $\ldots$ & $0 / 25(0)$ \\
\hline Cara cara & Peru & $0 / 37$ & $\ldots$ & $\ldots$ & $\ldots$ & $\ldots$ & $\ldots$ & $\ldots$ & $\ldots$ & $\ldots$ & $\ldots$ & $\ldots$ & $0 / 37(0)$ \\
\hline \multicolumn{14}{|l|}{ Blood orange } \\
\hline Tarocco & Italy & $5 / 97$ & $0 / 38$ & $\ldots$ & $\ldots$ & $\ldots$ & $\ldots$ & $\ldots$ & $\ldots$ & $\ldots$ & $0 / 9$ & $\ldots$ & $5 / 144(3.5)$ \\
\hline \multicolumn{14}{|c|}{ Common sweet orange } \\
\hline Hongjiangcheng & Auto & $\ldots$ & $\ldots$ & $\ldots$ & $\ldots$ & $\ldots$ & $\ldots$ & $16 / 30$ & $\ldots$ & $\ldots$ & $\ldots$ & $\ldots$ & $16 / 30(53.3)$ \\
\hline Bingtangcheng & Auto & $\ldots$ & $\ldots$ & $7 / 30$ & $\ldots$ & $\ldots$ & $\ldots$ & $\ldots$ & $\ldots$ & $\ldots$ & $14 / 51$ & $\ldots$ & $21 / 81(25.9)$ \\
\hline Unknown & Auto & $0 / 65$ & $\ldots$ & $3 / 20$ & $\ldots$ & $0 / 3$ & $0 / 9$ & $\ldots$ & $1 / 13$ & $\ldots$ & $0 / 12$ & $0 / 16$ & $4 / 138(2.9)$ \\
\hline \multicolumn{14}{|l|}{ Pummelo } \\
\hline Liangpingyou & Auto & $1 / 28$ & $\ldots$ & $\ldots$ & $\ldots$ & $\ldots$ & $\ldots$ & $\ldots$ & $\ldots$ & $\ldots$ & $\ldots$ & $\ldots$ & $1 / 28(3.6)$ \\
\hline Guanximiyou & Auto & $0 / 54$ & $\ldots$ & $\ldots$ & $2 / 11$ & $\ldots$ & $0 / 34$ & $\ldots$ & $1 / 26$ & $0 / 3$ & $11 / 49$ & $\ldots$ & $14 / 177(7.9)$ \\
\hline Shatianyou & Auto & $\ldots$ & $\ldots$ & $\ldots$ & $\ldots$ & $\ldots$ & $\ldots$ & $1 / 21$ & $\ldots$ & $0 / 19$ & $\ldots$ & $\ldots$ & $1 / 40(2.5)$ \\
\hline Gongshuibaiyou & Auto & $\begin{array}{l}\cdots \\
\cdots\end{array}$ & $\begin{array}{l}\cdots \\
\cdots\end{array}$ & $\begin{array}{l}\cdots \\
\ldots\end{array}$ & $\begin{array}{l}\cdots \\
\cdots\end{array}$ & $\begin{array}{l}\cdots \\
\cdots\end{array}$ & $\begin{array}{l}\cdots \\
\cdots\end{array}$ & $\ldots$ & $\begin{array}{l}\cdots \\
\cdots\end{array}$ & $\ldots$ & $\begin{array}{l}\cdots \\
\cdots\end{array}$ & $0 / 9$ & $0 / 9(0)$ \\
\hline Sour pummelo & Auto & $\ldots$ & $\ldots$ & $0 / 13$ & $\ldots$ & $\ldots$ & $\ldots$ & $\ldots$ & $0 / 3$ & $\ldots$ & $1 / 2$ & $\ldots$ & $1 / 18(5.6)$ \\
\hline \multicolumn{14}{|l|}{ Tangor } \\
\hline Shiranui & Jpn90 & $0 / 29$ & $\ldots$ & $\ldots$ & $\ldots$ & $\ldots$ & $\ldots$ & $\ldots$ & $\ldots$ & $\ldots$ & $\ldots$ & $\ldots$ & $0 / 29(0)$ \\
\hline Murcott & US70 & $2 / 11$ & $\ldots$ & $\ldots$ & $\ldots$ & $\ldots$ & $\ldots$ & $\ldots$ & $1 / 24$ & $\ldots$ & $\ldots$ & $\ldots$ & $3 / 35(8.6)$ \\
\hline Or & Israel & $2 / 26$ & $0 / 3$ & $\ldots$ & $\ldots$ & $\ldots$ & $\ldots$ & $\ldots$ & $3 / 20$ & $\ldots$ & $\ldots$ & $\ldots$ & $5 / 49(10.2)$ \\
\hline Unknown & Jpn90 & $0 / 9$ & $4 / 24$ & $\begin{array}{l}\cdots \\
\cdots\end{array}$ & $\begin{array}{l}\cdots \\
\cdots\end{array}$ & $0 / 4$ & $\begin{array}{l}\cdots \\
\cdots\end{array}$ & $\cdots$ & $0 / 2$ & $\begin{array}{l}\cdots \\
\cdots\end{array}$ & $\begin{array}{l}\cdots \\
\cdots\end{array}$ & $\cdots$ & $4 / 39(10.3)$ \\
\hline \multicolumn{14}{|l|}{ Kumquat } \\
\hline Chindou & Auto & $0 / 5$ & $\ldots$ & $\ldots$ & $\ldots$ & $\ldots$ & $\ldots$ & $\ldots$ & $\ldots$ & $\ldots$ & $0 / 2$ & $\ldots$ & $0 / 7(0)$ \\
\hline Luofu & Auto & $0 / 4$ & $\ldots$ & $\ldots$ & $\ldots$ & $\ldots$ & $\ldots$ & $\ldots$ & $\ldots$ & $\ldots$ & $0 / 4$ & $\ldots$ & $0 / 8(0)$ \\
\hline Bopijingan & Auto & $\ldots$ & $\cdots$ & $\cdots$ & $\cdots$ & $\cdots$ & $\cdots$ & $\cdots$ & $0 / 18$ & $\cdots$ & $\ldots$ & $\cdots$ & $0 / 18(0)$ \\
\hline \multicolumn{14}{|l|}{ Trifoliate } \\
\hline Zaoyangxiaoye & Auto & $2 / 12$ & $\ldots$ & $\ldots$ & $\ldots$ & $\ldots$ & $\ldots$ & $\ldots$ & $\ldots$ & $\ldots$ & $\ldots$ & $\ldots$ & 2/12 (16.7) \\
\hline Total & $\ldots$ & $65 / 770$ & $77 / 184$ & $59 / 275$ & $2 / 28$ & $0 / 76$ & $2 / 96$ & $50 / 182$ & $73 / 251$ & $65 / 221$ & $65 / 242$ & $0 / 25$ & $458 / 2,350(19.5)$ \\
\hline
\end{tabular}

a Detection rate of CYVCV in different provinces (Cho = Chongqing, Sic $=$ Sichuan, Yun = Yunnan, Gui = Guizhou, Zhe $=$ Zhejiang, Fuj $=$ Fujian, Gud $=$ Guangdong, Gux $=$ Guangxi, Jia $=$ Jiangxi, Hun $=$ Hunan, and Hub $=$ Hubei $)$ and number of orchards surveyed. Numerator $=$ number of plants infected and denominator $=$ number of test plants used.

${ }^{\mathrm{b}}$ Origin abbreviations: US50 $=$ imported from the United States in the 1930s, Auto $=$ autochthonous, Jpn50 $=$ imported from Japan in the $1950 \mathrm{~s}$, US70 $=$ imported from the United States in the 1970s, Peru = imported from Peru in the 1990s, Italy = imported from Italy in the 1970s, Israel = imported from Israel in the 2000s, and Jpn90 = imported from Japan in the 1990s. 
as shown by RT-PCR or PCR assays, strongly suggesting that the leaf symptoms on Or, Satsuma, Guanximiyou pummelo, and Shatangju were caused by CYVCV.

Furthermore, CYVCV did not develop apparent symptoms on Nanfengmiju, Ponkan, Jiaogan, Gonggan, Hongjiangcheng, Bingtangcheng, Newhall, 'Murcott' (C. reticulata Blanco $\times$ C. sinensis (L.) Osbeck), 'Shatianyou' pummelo (C. grandis (L.) Osbeck), 'Liangpingyou' pummelo (C. grandis (L.) Osbeck), sour pummelo (C. grandis (L.) Osbeck), and Zaoyangxiaoye trifoliate.

Characterization of the CYVCV genome. The genome size of CYVCV isolates collected from pummelo (GD-STP, GX-GXP, GZ-GXP, and HN-GXP), Ponkan (FJ-PK), Jiaogan (GD-JG), and Nanfengmiju (JX-NF) was 7,531 nucleotides (nt) (Table 1). However, the other 12 isolates, including SC-EL, SC-NH, CQ-PO, CQTA, YN-NH, YN-BTC. YN-EL, JX-NH, HN-STJ, GX-STJ, and GD-STJ, were 7,529 nt in length, with two bases ("-CA-") less at positions 28 and 29 in the $5^{\prime}$ untranslated region (UTR) region. Analysis of the genome sequences predicted six ORF on the positive strand, identifying a $5^{\prime}$ UTR and a $3^{\prime}$ UTR of $37 \mathrm{nt}$, followed by a poly(A) tail. The genome organization of all of these isolates and those published elsewhere was consistent (Cao et al. 2016; Loconsole et al. 2012a; Zhen et al. 2015). Alignment showed that all 5'UTR started with GAAAG and all six ORF of these CYVCV isolates started with an ATG codon and terminated with either a TAA stop codon (ORF1, -4 , and -5 ) or a TGA stop codon (ORF2, -3 , and -6).

Sequence alignments showed that the $19 \mathrm{CYVCV}$ isolates shared nucleotide sequence identities with ICRSV-K1 of 71.6 to $71.8 \%$ and with
ICRSV-Pu of 71.4 to $71.6 \%$ belonging to the same genus (Mandarivirus), and with PVX (the representative member of the same family Alphaflexiviridae) of less than 45.2\%, (Table 4). Furthermore, sequence analysis showed that these $19 \mathrm{CYVCV}$ isolates shared high nucleotide identities with four CYVCV isolates (KP313240 to KP313242 and JX040635) for the whole genome (97.1 to 99.8\%) (Table 4), 5'UTR (93.8 to $100 \%$ ), 3'UTR (100\%), and each of the six ORF (93.4 to $100 \%$ ). At the amino acid level, these $19 \mathrm{CYVCV}$ isolates also shared high identities with the same four CYVCV isolates for ORF1 (98.3 to 99.9\%), ORF2 (98.2 to $100 \%)$, ORF3 (97.2 to $100 \%$ ), ORF4 (96.8 to $100 \%$ ), ORF5 (96.6 to $100 \%$ ), and ORF6 (97.2 to $100 \%$ ).

Sequences of the REP and CP are some of the most conserved in the virus genome. As expected, all replication-associated domains were found in the polyprotein encoded by ORF1, including a viral methyltransferase at the $\mathrm{N}$-terminus (amino acids 39 to 329), an AlkB domain (amino acids 463 to 662), a viral helicase 1 in the central part (amino acids 926 to 1,157), and an RNA-dependent RNA polymerase 2 domain (amino acids 1,214 to 1,649). ORF5 encoded a putative $\mathrm{CP}$ containing a conserved domain (amino acids 140 to 278) with the characteristic motifs VWN (amino acids 226 to 228), PPANW (amino acids 236 to 240), and AAFDF (amino acids 252 to 256).

Genetic diversity and phylogenetic analyses. In phylogenetic trees derived from complete nucleotide sequences (Fig. 1) of available mandariviruses (ICRSV-K1 and ICRSV-Pu) and PVX, the representative member of the same family (Alphaflexiviridae), all of the CYVCV isolates grouped together as a sister branch of ICRSV-K1 and ICRSV-Pu, forming a distinct clade. All CYVCV isolates from

Table 4. Pairwise identity of nucleotide alignment of Citrus yellow vein clearing virus (CYVCV) genome nucleotide sequences ${ }^{\mathrm{a}}$

\begin{tabular}{|c|c|c|c|c|c|c|c|c|c|c|c|c|c|}
\hline $\operatorname{Ref}^{\mathbf{b}}$ & & 1 & 2 & 3 & 4 & 5 & 6 & 7 & 8 & 9 & 10 & 11 & 12 \\
\hline GZ-GXP & 1 & $\ldots$ & 18 & 24 & 18 & 44 & 19 & 17 & 13 & 34 & 53 & 24 & 24 \\
\hline JX-NF & 2 & 99.76 & $\ldots$ & 28 & 26 & 48 & 27 & 25 & 23 & 36 & 49 & 18 & 20 \\
\hline GD-STJ & 3 & 99.68 & 99.63 & $\ldots$ & 16 & 38 & 27 & 25 & 21 & 28 & 41 & 32 & 26 \\
\hline HN-STJ & 4 & 99.76 & 99.65 & 99.79 & $\ldots$ & 36 & 19 & 17 & 14 & 26 & 43 & 24 & 22 \\
\hline CQ-TA & 5 & 99.42 & 99.36 & 99.50 & 99.52 & $\ldots$ & 49 & 47 & 41 & 40 & 37 & 48 & 42 \\
\hline CYVCV-CQ & 6 & 99.75 & 99.64 & 99.64 & 99.75 & 99.35 & $\ldots$ & 12 & 15 & 35 & 56 & 25 & 19 \\
\hline GX-GXP & 7 & 99.77 & 99.67 & 99.67 & 99.77 & 99.38 & 99.84 & $\ldots$ & 11 & 33 & 54 & 23 & 15 \\
\hline FJ-PK & 8 & 99.83 & 99.69 & 99.72 & 99.81 & 99.46 & 99.80 & 99.85 & $\ldots$ & 30 & 48 & 22 & 20 \\
\hline GD-STP & 9 & 99.55 & 99.52 & 99.63 & 99.65 & 99.47 & 99.54 & 99.56 & 99.60 & $\ldots$ & 47 & 36 & 28 \\
\hline CQ-PO & 10 & 99.30 & 99.35 & 99.46 & 99.43 & 99.51 & 99.26 & 99.28 & 99.36 & 99.38 & $\ldots$ & 51 & 45 \\
\hline HN-GXP & 11 & 99.68 & 99.76 & 99.58 & 99.68 & 99.36 & 99.67 & 99.69 & 99.71 & 99.52 & 99.32 & $\ldots$ & 16 \\
\hline GD-JG & 12 & 99.68 & 99.73 & 99.65 & 99.71 & 99.44 & 99.75 & 99.80 & 99.73 & 99.63 & 99.40 & 99.79 & $\ldots$ \\
\hline JX-NH & 13 & 99.65 & 99.52 & 99.71 & 99.79 & 99.47 & 99.64 & 99.67 & 99.68 & 99.65 & 99.35 & 99.55 & 99.63 \\
\hline YN-BTC & 14 & 98.90 & 98.87 & 98.95 & 98.98 & 98.98 & 98.80 & 98.86 & 98.91 & 98.90 & 98.94 & 98.87 & 98.90 \\
\hline YN-EL & 15 & 98.84 & 98.87 & 98.92 & 98.96 & 98.92 & 98.79 & 98.84 & 98.91 & 98.88 & 98.94 & 98.86 & 98.88 \\
\hline GX-SA & 16 & 99.14 & 99.16 & 99.22 & 99.26 & 99.24 & 99.08 & 99.11 & 99.20 & 99.18 & 99.18 & 99.15 & 99.18 \\
\hline YN-NH & 17 & 98.86 & 98.83 & 98.91 & 98.94 & 98.94 & 98.77 & 98.84 & 98.87 & 98.86 & 98.84 & 98.83 & 98.88 \\
\hline SC-EL & 18 & 98.83 & 98.80 & 98.88 & 98.91 & 98.88 & 98.74 & 98.77 & 98.84 & 98.83 & 98.82 & 98.80 & 98.83 \\
\hline SC-NH & 19 & 98.80 & 98.78 & 98.86 & 98.88 & 98.83 & 98.74 & 98.77 & 98.83 & 98.80 & 98.76 & 98.78 & 98.80 \\
\hline GX-STJ & 20 & 99.15 & 99.20 & 99.28 & 99.23 & 99.28 & 99.08 & 99.14 & 99.16 & 99.15 & 99.30 & 99.15 & 99.23 \\
\hline CYVCV-YN & 21 & 98.78 & 98.70 & 98.83 & 98.90 & 98.82 & 98.75 & 98.77 & 98.86 & 98.74 & 98.69 & 98.74 & 98.71 \\
\hline CYVCV-PK & 22 & 97.57 & 97.54 & 97.68 & 97.62 & 97.54 & 97.58 & 97.56 & 97.58 & 97.54 & 97.61 & 97.54 & 97.57 \\
\hline CYVCV-Y1 & 23 & 97.40 & 97.48 & 97.42 & 97.45 & 97.38 & 97.28 & 97.30 & 97.38 & 97.33 & 97.61 & 97.42 & 97.46 \\
\hline ICRSV-K1 & 24 & 71.79 & 71.74 & 71.77 & 71.74 & 71.68 & 71.67 & 71.68 & 71.74 & 71.74 & 71.74 & 71.67 & 71.68 \\
\hline ICRSV-Pu & 25 & 71.61 & 71.55 & 71.62 & 71.58 & 71.49 & 71.51 & 71.53 & 71.58 & 71.58 & 71.64 & 71.49 & 71.54 \\
\hline PVX & 26 & 45.12 & 45.15 & 45.12 & 45.15 & 45.10 & 45.13 & 45.17 & 45.17 & 45.15 & 45.13 & 45.21 & 45.18 \\
\hline
\end{tabular}

\footnotetext{
a Lower values $=$ percent identity and upper values = total number of nucleotide differences.

b Accession numbers of reference sequences are as follows: SC-EL, CYVCV-CQ, YN-EL, and CYVCV-YN: CYVCV collected from Citrus limon Burm. f. in Sichuan (KX156748), Chongqing (KP313240), and Yunnan (KX156751 and KP313242), respectively; SC-NH, YN-NH, and JX-NH: CYVCV collected from C. sinensis (L.) Osbeck Newhall Navel in Sichuan (KX156749), Yunnan (KX156752), and Jiangxi (KX156747), respectively; HN-GXP, GX-GXP, and GZGXP: CYVCV collected from C. grandis (L.) Osbeck Guanximiyou in Hunan (KX156744), Guangxi (KX156741), and Guizhou (KX156743), respectively; HN-STJ, GX-STJ, and GD-STJ: CYVCV collected from C. reticulata Shatangju in Hunan (KX156745), Guangxi (KX156742), and Guangdong (KX156739), respectively; CQ-PO: CYVCV collected from Poncirus trifoliate Swingle var. Zaoyangxiaoye in Chongqing (KX156735); CQ-TA: CYVCV collected from C. sinensis (L.) Osbeck Tarocco in Chongqing (KX156736); JX-NF: CYVCV collected from C. reticulata Blanco Nanfengmiju in Jiangxi (KX156746); YNBTC: CYVCV collected from C. sinensis (L.) Osbeck Bingtangcheng in Yunnan (KX156750); GX-SA: CYVCV collected from C. unshiu Marc. in Guangxi (KX156734); GD-STP: CYVCV collected from C. grandis (L.) Osbeck Shatianyou in Guangdong (KX156740); GD-JG: CYVCV collected from C. tankan Hayata in Guangdong (KX156738); FJ-PK: CYVCV collected from C. reticulata Blanco in Fujian (KX156737); CYVCV-PK: CYVCV collected from C. sinensis (L.) Osbeck in Pakistan (KP313241); and CYVCV-Y1: CYVCV collected from C. limon Burm. f. in Turkey (JX040635). Indian citrus ringspot virus (ICRSV-KI, NC003093 and ICRSV-Pu, HQ324250) and Potato virus X (PVX, M72416).
} 
China were phylogenetically distinct from the isolates from Turkey and Pakistan, and clustered together in the same clade. Although CYVCV isolates from Yunnan and Sichuan Provinces were clustered in the same clade, other isolates from China did not show a relationship with the geographic origin of the sampled trees (Fig. 1). Moreover, according to the host species of the isolates, no clear clustering was observed in the phylogenetic trees generated by NJ, UPGMA, and PAUP* methods. Phylogenetic analyses of the REP and CP amino acid sequences recapitulated results seen for the complete nucleotide sequences.

CYVCV isolates were different at positions 482, 1,284, and 1,368 of the REP amino acid sequences. $\mathrm{P}^{482}$ was found in HN-GXP, GXGXP, and GZ-GXP, with moderate vein clearing on Guanximiyou pummelo. $\mathrm{M}^{1284}$ and $\mathrm{K}^{1368}$ were discovered in CYVCV isolates which caused severe yellow vein clearing, water soaking, and leaf distortion on Eureka lemon, whereas $\mathrm{R}^{482}, \mathrm{I}^{1284}$, and $\mathrm{R}^{1368}$ were shared by most CYVCV isolates without causing apparent symptoms in citrus plants. However, there was no clear correlation between the symptoms and $\mathrm{CP}$ gene or amino acid sequence variability of CYVCV isolates.

\section{Discussion}

CYVCV is an emerging citrus virus disease, having been first recognized in Yunnan Province of China during the past 8 years (Chen et al. 2014), but information on the presence of CYVCV from other citrus-producing provinces and other citrus cultivars was unknown. In this study, a large number of samples were collected from 11 major citrus-growing provinces of China for determining the incidence of
CYVCV. The results of this study showed that approximately $20 \%$ of the tested samples were infected with CYVCV, and that the virus is widespread in the Chinese major citrus-growing provinces. Furthermore, CYVCV spread rapidly in China. For instance, when CYVCV was first detected in Anyue County in 2010, hundreds of Eureka lemon plants tested positive for CYVCV. However, 4 years later, CYVCV were detected in most Eureka lemon orchards in Anyue County. The questions that arise from the present study are: Why is CYVCV spreading so quickly and how did CYVCV spread in China? Although CYVCV could be transmitted by A. craccivora and A. spiraecola from lemon to bean and from bean to bean very effectively (Önelge et al. 2011), until now no vector has been shown to transmit CYVCV from citrus to citrus (Loconsole et al. 2012a). Populations of Dialeurodes citri are numerous in all citrus-growing areas of China (Niu et al. 2014; Wang et al. 2013) and, in a recent study, CYVCV was detected in D. citri at high levels and, under trial conditions, CYVCV could be transmitted from sweet orange to sour orange by $D$. citri (data not shown). Therefore, D. citri is probably one of the important routes of CYVCV transmission in China. Further studies are required to identify the transmissibility of $D$. citri, and whether other vectors of CYVCV exist. The other possible explanation is that, presumably, CYVCV has been present in China for a long time but CYVCV is frequently latent in most citrus cultivars (Chen et al. 2015); therefore, the virus has never been noticed until the sensitive Eureka lemon was infected in Yunnan Province. Nevertheless, this speculation should be tested experimentally in a future study.

Table 4. (continued from preceding page)

\begin{tabular}{|c|c|c|c|c|c|c|c|c|c|c|c|c|c|}
\hline 13 & 14 & 15 & 16 & 17 & 18 & 19 & 20 & 21 & 22 & 23 & 24 & 25 & 26 \\
\hline 26 & 83 & 87 & 65 & 86 & 88 & 90 & 64 & 92 & 183 & 196 & 2146 & 2160 & 4517 \\
\hline 36 & 85 & 85 & 63 & 88 & 90 & 92 & 60 & 98 & 185 & 190 & 2150 & 2164 & 4515 \\
\hline 22 & 79 & 81 & 59 & 82 & 84 & 86 & 54 & 88 & 175 & 194 & 2147 & 2158 & 4517 \\
\hline 16 & 77 & 78 & 56 & 80 & 82 & 84 & 58 & 83 & 179 & 192 & 2149 & 2161 & 4515 \\
\hline 40 & 77 & 81 & 57 & 80 & 84 & 88 & 54 & 89 & 185 & 197 & 2154 & 2168 & 4519 \\
\hline 27 & 90 & 91 & 69 & 93 & 95 & 95 & 69 & 94 & 182 & 205 & 2155 & 2167 & 4516 \\
\hline 25 & 86 & 87 & 67 & 87 & 93 & 93 & 65 & 93 & 184 & 203 & 2154 & 2166 & 4513 \\
\hline 24 & 82 & 82 & 60 & 85 & 87 & 88 & 63 & 86 & 182 & 197 & 2150 & 2162 & 4513 \\
\hline 26 & 83 & 84 & 62 & 86 & 88 & 90 & 64 & 95 & 185 & 201 & 2150 & 2162 & 4515 \\
\hline 49 & 80 & 80 & 62 & 87 & 89 & 93 & 53 & 99 & 180 & 180 & 2149 & 2157 & 4516 \\
\hline 34 & 85 & 86 & 64 & 88 & 90 & 92 & 64 & 95 & 185 & 194 & 2155 & 2169 & 4510 \\
\hline 28 & 83 & 84 & 62 & 84 & 88 & 90 & 58 & 97 & 183 & 191 & 2154 & 2165 & 4512 \\
\hline$\ldots$ & 81 & 84 & 62 & 84 & 86 & 88 & 64 & 91 & 183 & 202 & 2151 & 2162 & 4520 \\
\hline 98.92 & $\ldots$ & 14 & 42 & 23 & 88 & 95 & 76 & 100 & 194 & 208 & 2161 & 2177 & 4522 \\
\hline 98.88 & 99.81 & $\ldots$ & 30 & 25 & 92 & 95 & 78 & 98 & 196 & 208 & 2163 & 2179 & 4517 \\
\hline 99.18 & 99.44 & 99.60 & $\ldots$ & 45 & 82 & 85 & 66 & 87 & 183 & 197 & 2158 & 2174 & 4513 \\
\hline 98.88 & 99.69 & 99.67 & 99.40 & $\ldots$ & 95 & 99 & 81 & 103 & 193 & 211 & 2151 & 2169 & 4523 \\
\hline 98.86 & 98.83 & 98.78 & 98.94 & 98.74 & $\ldots$ & 12 & 56 & 111 & 194 & 214 & 2151 & 2162 & 4520 \\
\hline 98.83 & 98.78 & 98.74 & 98.87 & 98.69 & 99.84 & $\ldots$ & 56 & 114 & 196 & 216 & 2152 & 2163 & 4523 \\
\hline 99.15 & 98.99 & 98.96 & 99.12 & 98.92 & 99.26 & 99.26 & $\ldots$ & 97 & 182 & 204 & 2143 & 2157 & 4520 \\
\hline 98.79 & 98.67 & 98.70 & 98.84 & 98.63 & 98.53 & 98.49 & 98.71 & $\ldots$ & 186 & 210 & 2157 & 2173 & 4520 \\
\hline 97.57 & 97.42 & 97.40 & 97.57 & 97.44 & 97.42 & 97.40 & 97.58 & 97.53 & $\ldots$ & 211 & 2157 & 2167 & 4519 \\
\hline 97.32 & 97.24 & 97.24 & 97.38 & 97.20 & 97.16 & 97.13 & 97.29 & 97.21 & 97.20 & $\ldots$ & 2163 & 2169 & 4532 \\
\hline 71.72 & 71.58 & 71.56 & 71.62 & 71.72 & 71.72 & 71.70 & 71.82 & 71.64 & 71.64 & 71.56 & $\ldots$ & 171 & 4818 \\
\hline 71.57 & 71.37 & 71.35 & 71.41 & 71.48 & 71.57 & 71.56 & 71.64 & 71.43 & 71.51 & 71.48 & 97.74 & $\ldots$ & 4812 \\
\hline 45.09 & 45.06 & 45.12 & 45.17 & 45.05 & 45.09 & 45.05 & 45.09 & 45.09 & 45.10 & 44.94 & 41.72 & 41.67 & $\ldots$ \\
\hline
\end{tabular}


Furthermore, even though a citrus virus-testing scheme has been implemented in China for decades, the evaluation of the virus status of budwood source trees in some nurseries, especially in small nurseries, is primarily based on visual inspection and the identification of characteristic symptoms (Zhou et al. 2013). However, CYVCV is frequently latent in most sweet orange, pummelo, tangerine, and mandarin varieties (Chen et al. 2015) and, therefore, this "virus testing" approach allows for the dissemination of CYVCV. In this study, we found some new CYVCV cases occurred in Chongqing in 2016, because growers imported CYVCV-infected Eureka lemon plants from areas such as Anyue County with a high incidence of CYVCV. The results indicated that, although $D$. citri transmission may be responsible for local spread, CYVCV dispersal to new areas or countries occurred by graft propagation of virus-infected plant tissues, as seen in other insect-transmitted plant viruses such as CTV (Bar-Joseph et al. 1989) and CCDaV (Loconsole et al. 2012b). The results also highlight how the propagation of infected plant material might exacerbate the dissemination of CYVCV. This illustrates the need to implement programs for the production of certified citrus propagating material in order to prevent further spread of the virus.

It is known that the $5^{\prime} \mathrm{UTR}$ region plays an important role in gene expression (Alvarez et al. 2008; Sullivan and Ganem 2005). Interestingly, seven CYVCV isolates used in this study as well as CYVCV isolates (KP313240 and NC026592) previously reported from China possessed a 2-nt insertion (-CA-) at positions 28 and 29 in the $5^{\prime} \mathrm{UTR}$ region compared with the other CYVCV isolates. Whether this 2-nt insertion has some effect in viral function such as movement, transmissibility, or pathogenicity is unknown.
In this study, sequence analysis showed that $19 \mathrm{CYVCV}$ isolates from China shared high nucleotide identity with four CYVCV isolates which were deposited in GenBank for the whole genome, $5^{\prime}$ UTR, 3'UTR, and each of six ORF. In a previous study, Zhen et al. (2015) speculated that the sequence of CYVCV was stable based on the sequence analysis on two full sequences of CYVCV isolated from lemon in China and Turkey. Another possible explanation for the lack of sequence heterogeneity is that the introduction of CYVCV into China is recent and its subsequent dispersion has been rapid. A similar phenomenon was also found for Citrus leaf blotch virus (Vives et al. 2002). Phylogenetic analysis was also implemented to investigate the evolutionary relationships among CYVCV isolates from different countries. The result revealed that CYVCV isolates from China were clearly distinct from the isolates $\mathrm{Y} 1$ and PK which have been collected from Turkey and Pakistan, indicating that CYVCV may have different points of origin. Furthermore, the phylogenetic tree separated all of the CYVCV isolates from China into the same group, without any clear clustering according to host species or geographic origin. In previous studies, isolates of Apple stem pitting virus and Apple chlorotic leaf spot virus from different geographical origins and hosts were grouped into the same clade, indicating the important role of the exchange of infected plant material on virus dissemination (Liu et al. 2013; Mathioudakis et al. 2010). Our results also indicate that the movement of vegetative propagative materials or grafting of infected plants was also probably one of the important routes of CYVCV transmission in China.

In conclusion, the results of this study revealed the high occurrence and very low level of nucleotide sequence diversity of CYVCV

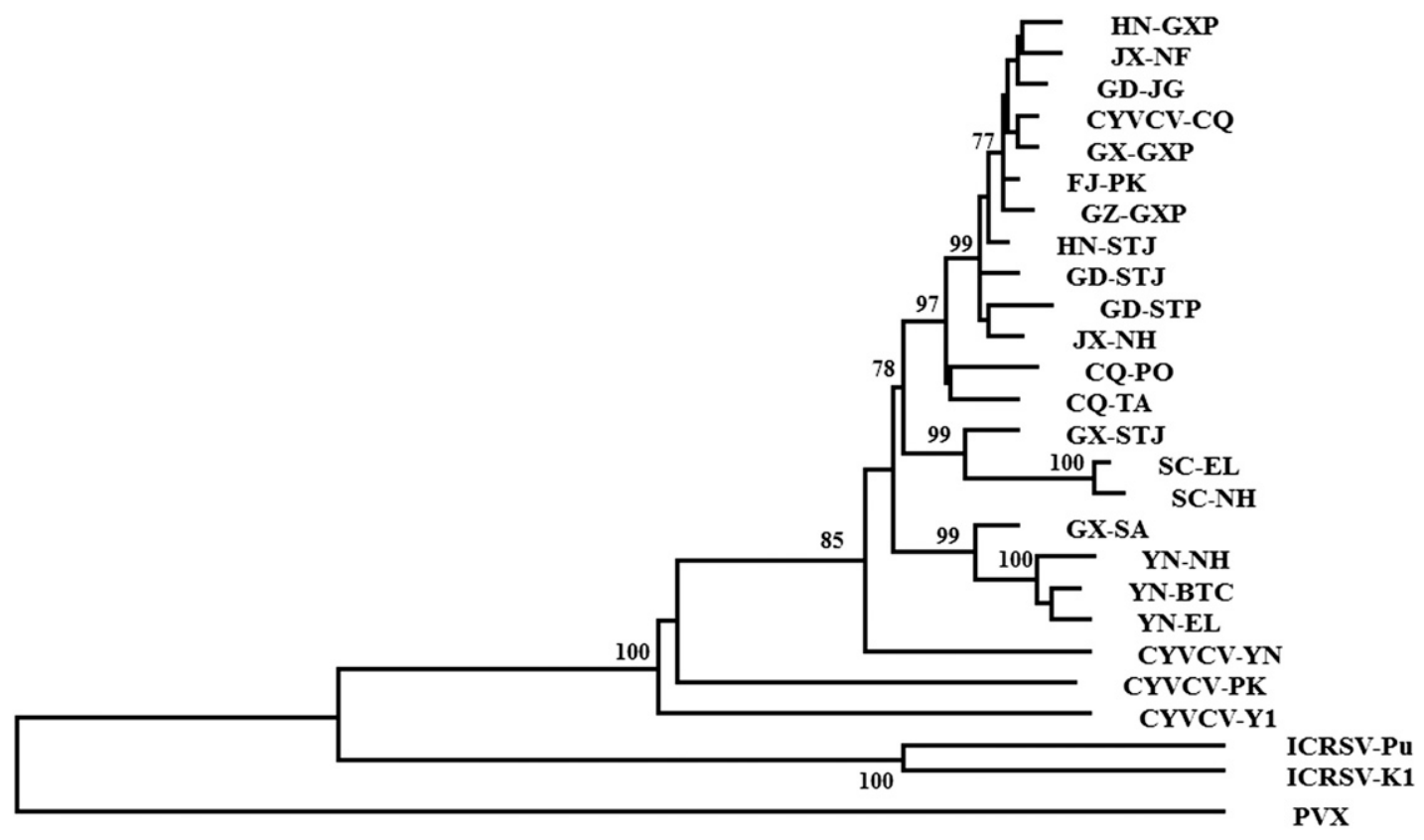

Fig. 1. Phylogenetic tree generated by the neighbor-joining method from the alignment of the genome nucleotide sequences of Citrus yellow vein clearing virus (CYVCV) and members belonging to the same genus, Mandarivirus (Indian citrus ringspot virus, ICRSV), and the same family, Alphaflexiviridae (Potato virus $X$, PVX), respectively, using MEGA (version 6.0). Bootstrap values for 10,000 replicates are indicated at the main branches. Branch length is proportional to number of nucleotide changes (bar). Accession numbers of reference sequences are as follows: SC-EL, CYVCV-CQ, YN-EL, and CYVCV-YN: CYVCV collected from Citrus limon Burm. f. in Sichuan (KX156748), Chongqing (KP313240), and Yunnan (KX156751, KP313242), respectively; SC-NH, YN-NH, and JX-NH: CYVCV collected from C. sinensis (L.) Osbeck Newhall navel in Sichuan (KX156749), Yunnan (KX156752), and Jiangxi (KX156747), respectively; HN-GXP, GX-GXP, and GZ-GXP: CYVCV collected from C. grandis (L.) Osbeck Guanximiyou in Hunan (KX156744), Guangxi (KX156741), and Guizhou (KX156743), respectively; HN-STJ, GX-STJ, and GD-STJ: CYVCV collected from C. reticulata Shatangju in Hunan (KX156745), Guangxi (KX156742), and Guangdong (KX156739), respectively; CQ-PO: CYVCV collected from Poncirus trifoliate Swingle var. Zaoyangxiaoye in Chongqing (KX156735); CQ-TA: CYVCV collected from C. sinensis (L.) Osbeck Tarocco in Chongqing (KX156736); JX-NF: CYVCV collected from C. reticulata Blanco Nanfengmiju in Jiangxi (KX156746); YN-BTC: CYVCV collected from C. sinensis (L.) Osbeck Bingtangcheng in Yunnan (KX156750); GX-SA: CYVCV collected from C. unshiu Marc. in Guangxi (KX156734); GD-STP: CYVCV collected from C. grandis (L.) Osbeck Shatianyou in Guangdong (KX156740); GD-JG: CYVCV collected from C. tankan Hayata in Guangdong (KX156738); FJ-PK: CYVCV collected from C. reticulata Blanco in Fujian (KX156737); CYVCV-PK: CYVCV collected from C. sinensis (L.) Osbeck in Pakistan (KP313241), CYVCV-Y1: and CYVCV collected from C. limon Burm. f. in Turkey (JX040635). ICRSV-KI (NC003093), ICRSV-Pu, (HQ324250), and PVX (M72416). 
in China. This finding significantly expands the known geographic distribution of CYVCV in China and provides new genomic information for understanding virus epidemiology and evolution.

\section{Acknowledgments}

This work was partially supported by Fundamental Research Funds for the Central Universities (XDJK2015A009), Two Rivers Scholar and Chongqing Research Program of Basic Research, and Frontier Technology (cstc2015jcyjBX0043).

\section{Literature Cited}

Alshami, A. A. A., Ahlawat, Y. S., and Pant, R. P. 2003. A hitherto unreported yellow vein clearing disease of citrus in India and its viral aetiology. Indian Phytopathol. 56:422-427.

Alvarez, D. E., Filomatori, C. V., and Gamarnik, A. V. 2008. Functional analysis of dengue virus cyclization sequences located at the $5^{\prime}$ and $3^{\prime}$ UTRs. Virology 375:223-235.

Bar-Joseph, M., Marcus, R., and Lee, R. F. 1989. The continuous challenge of citrus tristeza virus control. Annu. Rev. Phytopathol. 27:291-316.

Cao, M. J., Wu, Q., Atta, C. S., Su, H. N., Yu, Y. Q., Chen, H. M., and Zhou, C. Y. 2016. First molecular evidence of Citrus yellow vein clearing virus from citrus in Punjab, Pakistan. Plant Dis. 100:540.

Catara, A., Azzaro, A., Davino, M., and Polizzi, G. 1992. Yellow vein clearing of lemon in Pakistan. Pages 364-367 in: 12th Conf. Int. Organ. Citrus Virol. New Delhi, India. P. Moreno, J. V. da Graca, and L. W. Timmer, eds. International Organization of Citrus Virologists, Riverside, CA.

Chen, H. M., Li, Z. A., Wang, X. F., Zhou, Y., Tang, K. Z., Zhou, C. Y., Zhao, X. Y., and Yue, J. Q. 2014. First report of Citrus yellow vein clearing virus on Lemon in Yunnan, China. Plant Dis. 98:1747.

Chen, H. M., Wang, X. F., Zhou, Y., Zhou, C. Y., Guo, J., and Li, Z. A. 2015. Biological characterization and RT-PCR detection of a new disease of Eureka lemon. J. Plant Prot. 42:557-563.

Ito, T., Ieki, H., and Ozaki, K. 2002. Simultaneous detection of six citrus viroids and Apple stem grooving virus from citrus plants by multiplex reverse transcription polymerase chain reaction. J. Virol. Methods 106:235-239.

Iwanami, T., Kondo, Y., Makita, Y., Azeyanagi, C., and Ieki, H. 1998. The nucleotide sequence of the coat protein genes of satsuma dwarf virus and navel orange infectious mottling virus. Arch. Virol. 143:405-412.

Liu, P., Zhang, L., Zhang, H. W., Jiao, H. H., and Wu, Y. F. 2013. Detection and molecular variability of Apple stem grooving virus in Shaanxi, China. J. Phytopathol. 161:445-449.

Loconsole, G., Önelge, N., Potere, O., Giampetruzzi, A., Bozan, O., Satar, S., De Stradis, A., Savino, V., Yokomi, R. K., and Saponari, M. 2012a. Identification and characterization of Citrus yellow vein clearing virus, a putative new member of the genus Mandarivirus. Phytopathology 102:1168-1175.

Loconsole, G., Saldarelli, P., Doddapaneni, H., Savino, V., Martelli, G. P., and Saponari, M. 2012b. Identification of a single-stranded DNA virus associated with citrus chlorotic dwarf disease, a new member in the family Geminiviridae. Virology 432:162-172.

Mathioudakis, M. M., Maliogka, V. I., Katsiani, A. T., and Katis, N. I. 2010. Incidence and molecular variability of Apple stem pitting and Apple chlorotic leaf spot viruses in apple and pear orchards in Greece. J. Plant Pathol. 92:139-147.

Niu, J. Z., Helen, H. S., Zhang, Y. X., Lin, J. Z., Dou, W., and Wang, J. J. 2014. Biological control of arthropod pests in citrus orchards in China. Biol. Control 68:15-22.

Önelge, N. 2002. First report of yellow vein clearing of lemons in Turkey. J. Turk Phytopathol. 32:53-55

Önelge, N., Satar, S., Elibüyük, Ö., Bozan, O., and Kamberoğlu, M. 2011. Transmission studies on Citrus yellow vein clearing virus. Pages 11-14 in: 18th IOCV Conf. Brazil. J. V. da Graca, R. F. Lee, and R. K. Yokomi, eds. International Organization of Citrus Virologists, Riverside, CA.

Roy, A., Fayad, A., Barthe, G., and Brlansky, R. H. 2005. A multiplex polymerase chain reaction method for reliable, sensitive and simultaneous detection of multiple viruses in citrus trees. J. Virol. Methods 129:47-55.

Sullivan, C. S., and Ganem, D. 2005. MicroRNAs and viral infection. Mol. Cell 20:3-7.

Tamura, K., Stecher, G., Peterson, D., Filipski, A., and Kumar, S. 2013. MEGA6: Molecular evolutionary genetics analysis version 6.0. Mol. Biol. Evol. 30:2725-2729.

Vives, M. C., Rubio, L., Galipienso, L., Navarro, L., Moreno, P., and Guerri, J. 2002. Low genetic variation between isolates of Citrus leaf blotch virus from different host species and different geographical origins. J. Gen. Virol. 83: 2587-2591.

Vives, M. C., Velázquez, K., Pina, J. A., Moreno, P., Guerri, J., and Navarro, L. 2013. Identification of a new Enamovirus associated with citrus vein enation disease by deep sequencing of small RNAs. Phytopathology 103:1077-1086.

Wang, P. P., Song, X. H., and Zhang, H. Y. 2013. Isolation and characterization of Aschersonia placenta from citrus orchards and its pathogenicity towards Dialeurodes citri (Ashmead). J. Invertebr. Pathol. 112:122-128.

Zhen, S., Kurth, E. G., Peremyslov, V. V., Zhou, C. Y., and Dolja, V. V. 2015 Molecular characterization of a Citrus yellow vein clearing virus strain from China. Arch. Virol. 160:1811-1813.

Zhou, C. Y., Wang, X. F., Zhou, Y., and Liu, J. X. 2013. Occurrence and control citrus viral diseases in China. China Fruit News 30:70-72.

Zhou, Y., Chen, H. M., Wang, X. F., Li, Z. A., Tang, M., and Zhou, C. Y. 2015 Lack of evidence for seed transmission of Citrus yellow vein clearing virus despite its frequent detection in seed tissues. J. Plant Pathol. 97:1-3.

Zhou, Y., Chen, H. M., Wang, X. F., Li, Z. A., and Zhou, C. Y. 2016. Development and application of nested RT-PCR assay for detection of Citrus yellow vein clearing virus. J. Plant Prot. 43:255-259. 